\title{
Smoothing of MPEG Multi-program Video Coding for Packet Networks
}

Luis Teixeira ${ }^{(*)(+)}$, Teresa Andrade ${ }^{(")}($ (*)

(*) INESC-Largo Mompilher 22, 4000 Porto Portugal

(+) Universidade Católica Portuguesa, Rua Diogo Botelho 1327, 4150 Porto, Portugal

(\#) Faculdade de Engenharia da U.P., Rua dos Bragas 4099 Porto Codex, Portugal

\begin{abstract}
In TV broadcast applications, the use of MPEG2 standard allows economies in transmission bandwidth while providing a service with a quality similar or better to the current analogue systems. Interframe and intraframe techniques are used resulting in VBR bit streams, if constant quality is aimed. The number of bits depends on the motion and spatial detail of the video sequences. The compressed picture size differs by a factor up to 8 or more. Different TV programmes present different bandwidth requirements depending on the type of scene being transmitted. Thus buffering is needed to smooth rate fluctuations of encoder's output between pictures. Without smoothing, performance of networks carrying such video traffic can be compromised. The use of dynamic multiplexing schemes to jointly transmit different programmes allows a more efficient distribution of the available channel bandwidth by instantaneously assigning it among video sources according to their needs. The ATM technology provides the mechanisms for flexible allocation of resources and statistical transmission of VBR traffic, while providing the necessary quality of service guarantees to meet the real-time constraints of TV distribution services. This paper proposes the use of dynamic multiplexing algorithms at the user terminal to transmit a number of MPEG2 VBR encoded TV programmes over a VBR ATM channel. It addresses the problem of jointly controlling the channel and several VBR video sources bit rates.
\end{abstract}

Key Words: MPEG 2, ATM, dynamic multiplexing, rate control

\section{Introduction}

Video compression algorithms are able to reduce the number of bits necessary to represent a given sequence, by exploiting spatial and temporal redundancy in the original video sequences. Because this redundancy is variable (due to different degrees of spatial detail and amount of movement present in each picture or sequence), so will be the number of bits generated by the algorithm to achieve a given picture quality level for the recovered video sequence. This means that compression algorithms such as the MPEG2 standard will generate Variable Bit Rate (VBR) encoded bit streams if constant quality is to be obtained. Likewise, a constant bit rate compressed bit stream will reproduce a video sequence presenting a variable picture quality.

A digital TV supplier broadcasting simultaneously several different TV programmes using a constant bit rate transmission channel, will either perform a deterministic distribution of the transmission capacity or will dynamically assign it among all video sources by using dynamic multiplexing schemes. However, in both situations, the total amount of transmission bandwidth will always be kept constant and equal to the maximum channel capacity, regardless of the effective bit rate instantaneously required by the video sources. This means that it would be possible to use VBR video encoding algorithms in each source, but the permissible range of variation of bit rate of each source would be rather constrained.

Transmission networks using the Asynchronous Transfer Mode (ATM) technology, provide the mechanisms for flexible/dynamic allocation of resources, thus allowing 
the transmission of VBR traffic while providing quality of service guarantees to meet the real-time constraints of TV distribution services. Dynamic multiplexing schemes are able to instantaneously assign the available transmission resources among video sources according to their instantaneous needs, thus meeting the statistical characteristics of bandwidth-on-demand and switching capabilities provided by the ATM technique. This allows the use of VBR video encoding algorithms and consequently the possibility for the TV supplier to offer a constant or near-constant quality service with bandwidth economies. Considering that independent video sources are highly de-correlated and that the number of bits produced in each source will be variable, it will be possible to re-use in other sources, bandwidth made available by one source in periods of low activity. This means that $n$ video sources each requiring independently a CBR channel of bandwidth $R$ bits/s, might be transmitted together over one single channel with bit rate less than $R^{*} n$. This reduction in capacity is known as the Statistical Multiplexing Gain (SMG). The SMG can be obtained in the network through the use of the ATM technology and setting up $n$ independent VBR channels for each video source. It can also be achieved directly at the user terminal equipment by requesting one single VBR channel with average bit rate less than $n^{*} R$ for all the $n$ sources and dynamically multiplexing them before accessing the ATM network. Performing statistical multiplexing at both levels - at the user terminal equipment and in the network - may prove to be interesting for some applications such as distribution of a number of TV programmes. It will benefit from the advantages already reported in previous experiments and will allow the $T V$ supplier to distribute the negotiated bandwidth among the different sources in order to maintain a certain level of quality for all of them while reducing the total required bandwidth.

\section{Coding Issues}

Numerous works have outlined the advantages of variable bit rate (VBR) transmission of video over standard constant bit rate (CBR) methods [1]. Transmission of VBR video over ATM networks offers advantages in terms of both video quality and network utilization. In the literature, e.g. [2,3], VBR compression is claimed to be $30 \%$ more efficient in bit rate than CBR compression. Video coders operating at constant perceptual quality produce variable rate output; thus, VBR in principle offers the advantage of transmitting the video without requiring buffering at the encoder.

Estimates of video quality and multiplexing gain are usually based on simple scenarios, and in general have considered separately networking and source coding (e.g., assuming that constant quantizer bit rate traces are representative of VBR video transmission independently of the type of networking environment considered). However, the key to successful implementation of VBR video transmission lies in the interface between video and network, specifically in the rules used to determine the bit rate that can be allowed into the network from each source. Once we have specified the interface we can produce models for the video bit rate under the chosen set of rate constraints and use these models to estimate network utilization. Generally, studies of statistical multiplexing focus on the prediction of the probability of packet loss as a function of, for instance, the buffer size, the number of users of the network, etc. Since the probability of packet loss must be kept small it is not usually possible to use the network to its full capacity. Moreover, the fact that the results of these studies depend 
strongly on the employed statistical models of the video sources implies the risk that the actual network performance will be significantly different from the theoretical performance if the actual source behavior deviates from the employed statistical model. In our work, the different encoders share the same bit rate control. This joint bit rate control allocates bits to each program in such a way that the overall bit rates sum meet the negotiated connection parameters. Techniques are being developed to allow an optimal transcoding process. These techniques will remove the need for the encoders to be physically close to one another. In order to better study and classify our results we have characterised video sources by means of the picture complexity. A measure of this parameter is obtained by computing the average value of spatial local activity. The complexity of the scene being coded can be estimated from the complexity of the different frame types in the GOP: the spatial complexity from the intraframes (I frame) and the motion, which determines the complexity of the P and B frames (interframe coding). The sequences being used in the simulations are divided according to their content complexity (table 1), measured through the mean and the variance values of the local activity.

\begin{tabular}{|c|l|}
\hline Class & Content Complexity \\
\hline A & low spatial detail \& low amount of movement \\
\hline B & Medium spatial detail \& low amount of movement or vice versa \\
\hline C & High spatial detail \& medium amount of movement or vice versa \\
\hline
\end{tabular}

Table 1 - Classification regarding content complexity

\subsection{Dynamic Bandwidth Allocation Algorithm}

The dynamic bandwidth allocation algorithm, used to the moment, consists in 4 steps and is applied during a picture period. In the first step, the reference bandwidth (BWref) of each video source is determined based on the total available transmission bandwidth, the picture coding complexity and type, GOP structure of each video source and the current state of the total virtual buffer.

$$
\begin{aligned}
& B W_{\text {ref } \_t}=\frac{R \times X_{i}}{X_{i}+\frac{N_{P} \times X_{p}}{K_{p}}+\frac{N_{b} \times X_{i}}{K_{b}}} \\
& B W_{\text {ref } \_P}=\frac{R \times \frac{X_{p}}{K_{p}}}{X_{i}+\frac{N_{P} \times X_{p}}{K_{p}}+\frac{N_{b} \times X_{i}}{K_{b}}} \\
& B W_{\text {ref } B}=\frac{R \times \frac{X_{b}}{K_{b}}}{X_{i}+\frac{N_{P} \times X_{p}}{K_{p}}+\frac{N_{b} \times X_{i}}{K_{b}}}
\end{aligned}
$$

$B W_{r e f_{-} i}, B W_{r e f_{-} P}$ and $B W_{r e f_{-} B}$ are bandwidth for $1, P$ and $B$ pictures for a video source within a GOP. $K_{P}=1.0$ and $K_{b}=1.4$ are constants dependent on the 
quantisation matrices; $N_{p}$ and $N_{b}$ are the numbers of $\mathrm{P}$ pictures and $\mathrm{B}$ pictures remaining in the current GOP in the encoding order; $R$ is the transmission bandwidth allocate to the channel during one GOP; $N_{\text {Gop }}$ is the total number of pictures in the GOP; bit_rate is the ratio between the total available transmission bandwidth and the number of sources; $X$ variables are the "global complexity measure" of the different pictures types. They are updated by calculating the product of the number of bits generated by encoding a picture and the average quantisation parameter (computed with the actual quantisation values used during the encoding of all macroblocks, including the skipped ones) for each of the different pictures types.

In the second step, the estimated bandwidth is determined for the optimal distribution of the total available transmission bandwidth according to picture coding type and video source complexity. In the third step, the available bandwidth is allocated to each video source by considering the estimated bandwidth. Finally, in the last step, a reference value for the quantisation parameter is determined for the picture. The reference value of the quantisation parameter is then independently modulated in each video encoder according to the spatial activity in the macroblock to obtain the value of the quantisation parameter, mquant, that is used to quantise each macroblock.

\subsection{Video Source Modelling}

A rate-distortion model of the video encoder is being studied. We are analysing the relation between step size, bit rate and image quality. Preliminary results are presented for open-loop (fixed quantiser step size) simulations of several sequences and 1.25 Mbps CBR simulations using the TM5 rate control mechanism. A linear regression was used to match obtained results with an exponential curve for each sequence.

The sequences that were used are SIF sequences and we are using mquant linear scale. $(2,10,16,20,26,30,36,40,46,50,56,60,62)$. The closed loop values are also presented and were placed in the chart according to the average value of mquant. In closed loop the bitrates were of 1250000 bits /s.

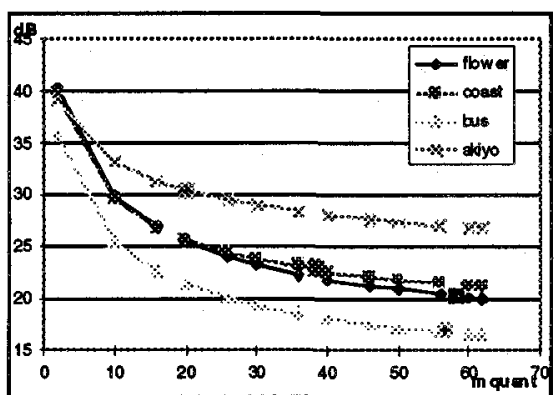

Fig. 1.a) Average SNR/ MB

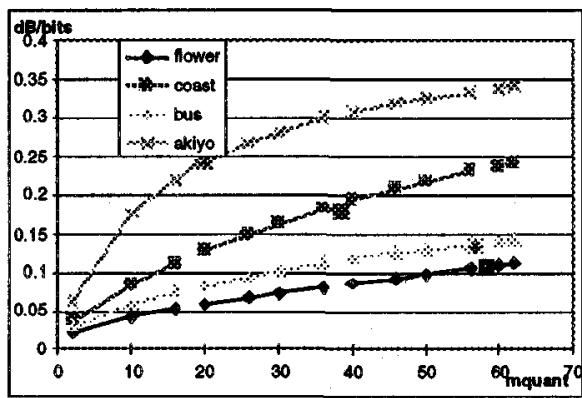

Fig 1.b) (Average SNR)/(average $n^{\circ}$ bit)/ MB 


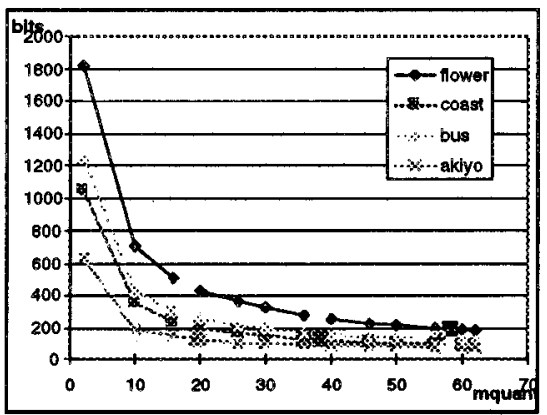

Fig. 1 c) Average $n^{\circ}$ bit / MB

The standard deviation referring to bit usage is higher when simulations are done with low values of mquant, decreasing exponentially. For SNR, the standard deviation increases with mquant. In closed-loop deviation of SNR is significantly higher when compared to the values obtained in open loop with similar values of mquant. Using a linear regression we have obtained the following curves of bit usage and SNR:

\begin{tabular}{|l|c|c|c|c|}
\hline Sequence & Bit usage & Correlation & SNR & Correlation \\
\hline Flower Garden & $3227 m q p^{-0.6892}$ & -0.996 & $47.4 m q{ }_{1} p^{-0.2090}$ & -0.999 \\
\hline Coast Guard & $1799 m q p^{-0.7351}$ & -0.999 & $45.0 m q p^{-0.1850}$ & -0.999 \\
\hline Bus & $2096 m q p^{-0.7042}$ & -0.999 & $42.5 m q p^{-0.2303}$ & -0.999 \\
\hline Akiyo & $812 m q p^{-0.5897}$ & -0.988 & $42.6 m q p^{-0.1122}$ & -0.999 \\
\hline
\end{tabular}

Table $2-$ Bit usage and SNR

\subsection{Instantaneous changes in bit rate}

Some simulations were made in order to observe the evolution of the encoder buffer and decoder buffer occupancy. The coastguard sequence was used and was encoded in CBR mode with the following parameters: GOP $=12$; vbv_buffer_size $=18 * 16384$ $=294912$; frame_rate $=25 \mathrm{fps}$.

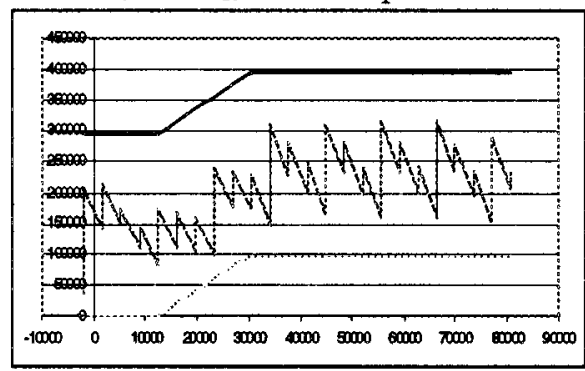

Fig. 2.a) encoder buffer occupancy

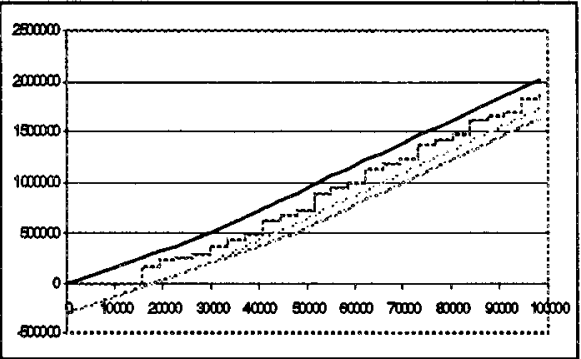

Fig. 2.b) address decoder buffer evolution

Figure 2 presents the results ( $\mathrm{x}$ axis corresponds to ticks of $90 \mathrm{kHz}$ ). In this simulation, the bit rate was changed from 1500 to $2000 \mathrm{kbps}$ (an increase in the encoder buffer).

\section{User-Network Interface}

ATM networks provide a certain Quality of Service (QOS) guarantees associated with defined classes of service. The QOS parameters (such as maximum admissible delay, 
Cell Delay Variation and Cell Loss Ratio) as well user traffic characteristics (such as average cell rate, peak cell rate, burstiness, peak duration and source type) are negotiated at connection set-up (though may also be re-negotiated during the call). As long as the user does not violate the traffic contract, the network will always provide a service with the requested QOS. In order to maintain the agreed QOS of all ATM connections, the network will be constantly performing a set of traffic control operations. This mechanism will be able to prevent that traffic violations at a given connection affect the QOS of other connections. When a given user violates the traffic contract, the network will no longer have the responsibility of maintaining the agreed QOS. It is therefore the interest of the user not to exceed the values negotiated in the traffic contract. This means that the user will have also to implement some kind of policing functions at the user-network interface, in order to ensure that the negotiated bandwidth will be respected and consequently that the agreed QOS guarantees will be maintained.

At connection set-up, the user negotiates a traffic contract defined by a traffic descriptor and QOS parameters. The traffic descriptor consists in a generic list of traffic parameters to describe the characteristics of the source traffic. In our experiments we consider the peak cell rate and average cell rate. The peak cell rate is a mandatory parameter to be defined in the traffic contract. It specifies the upper limit of the source traffic that can be submitted to the ATM connection. Peak cell rate is defined in terms of the minimum time $T$ between two consecutive requests in the user terminal equipment to send a packet of data to the network (i.e. to send an ATMPacket Data Unit). To perform traffic control policies by monitoring the peak rate cell, it is necessary to specify also the maximum cell delay variation (CDV) tolerated by the connection. A possible algorithm implemented by the network at the user-network interface to perform peak cell tate conformance of an ATM connection taking into account the CDV tolerance of the connection, is the Leaky Bucket (LB) algorithm. The $L B$ capacity is measured in units of $L B=T+\delta$, where $T$ is the inverse of the peak cell rate and $\delta$ the maximum tolerated CDV.

In this study, different video channels are aggregated in a single MPEG2 Multi Program Transport Stream (MPTS) before accessing the network. Those video channels are multiplexed using the dynamic multiplexing algorithm described in the previous section. The algorithm distributes up to the total available bandwidth among all channels, in such a way as to maintain constant the picture quality in every channel using the smallest possible amount of bandwidth There is only one network interface (NI) and only one connection to be established. The NI negotiates the parameters for the initial connection based upon traffic characteristics of the multiplexed stream. Those parameters may be re-negotiated during the call with a certain periodicity, if the dynamic multiplexer-controller/source-modelling block informs the NI that more or less bandwidth is required to achieve the proposed level of quality for all channels. The NI is constantly monitoring the multiplexed bit stream rate in order to be able to either re-negotiate the connection parameters or to inform the multiplexer controller that it must decrease its output bit rate. 


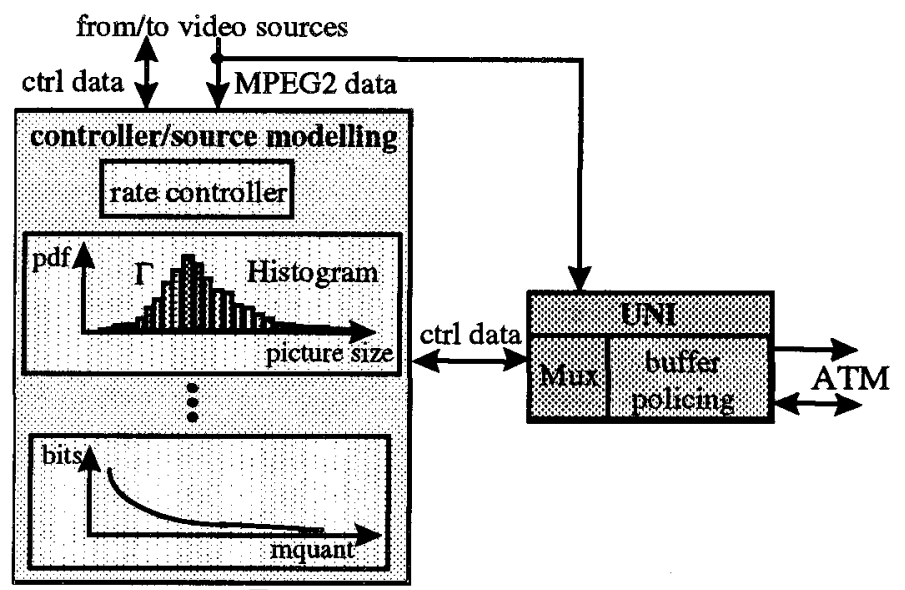

Fig. 3 Network Interface Model

\section{Summary}

The goal of the work described in this paper is to develop dynamic multiplexing algorithms to be implemented at the user terminal equipment and to study the potential benefits of jointly controlling the bit rate of several encoders and the channel bit rate. The bit rate of the VBR transmission channel will be constrained by leaky bucket policing functions. The source bit rate will be determined and controlled on a picture basis by the proposed dynamic bandwidth allocation algorithm with the objective of maintaining a minimum level of quality among all video sources using always the least possible bandwidth and without violating the traffic contract.

\section{Acknowledgements}

This work was supported by the Junta Nacional Cientifica e Tecnologica (grant PRAXIS-XXI /4/4.1/BD/2256).

\section{References}

[1] W. Verbiest, L. Pinnoo, and B. Voeten, "The impact of the ATM concept on video coding," The IEEE Journal on Selected Areas in Communications, vol. 6, pp. 1623, December 1988.

[2] Toshio Koga, Yukishiko Iijima. Kazumoto linuma, Tatsuo Ishiguro,"Statistical performance analisys of an interframe encoder for broadcast television signals", IEEE Trans. On Comm., vol. 29, $\mathrm{n}^{\circ} 12$, pp 1868-1875, Dec. 1981.

[3] Willem Verbiest, Luc Pinnoo, "A variable bit rate video codec for ATM networks", IEEE Journ. On Sel. Areas in Comm., vol. 7, pp 761-770, June 1989.

[4] Proceedings of the $6^{\text {th }}$ International Workshop on Packet Video, September 1994.

[5] L.Teixeira, T. Andrade, "Dynamic Bandwidth Allocation for a MPEG2 MultiEncoder Video System", Symposium on Advanced Imaging and Network Technologies, Berlin, October 1996.

[6] M. Schwartz, "Broadband Integrated Networks", Prentice Hall, 1996. 